\title{
Layout optimization of fare gates in rail transit station based on pedestrian route choice
}

\author{
Zihan Sun ${ }^{1, a}, \mathrm{Xu} \mathrm{Wu}{ }^{1, b}$ \\ ${ }^{1}$ School of Traffic and Transportation, Beijing Jiaotong University, Beijing 100044, China \\ asunzihannuli@163.com, ${ }^{b}$ wuxu@bjtu.edu.cn
}

\begin{abstract}
Keywords: route choice, automatic fare gate, layout optimization, simulation.
Abstract. The layout of fare gates have become one of the most significant bottleneck in streamline organization. In actual practice, the layout principle and combining scheme are not clearly demonstrated. Here, we build a multistage pedestrian-and-gate route choice model on account of time cost based on research on the microcosmic characteristics of the behavior of pedestrian in rail transit station as well as the temporal and spatial characteristics of the passenger flow. We further investigate the corresponding algorithm which can convert the time cost to the selection probability and decide which to choose according to the probability based on the random number method. Two indexes are selected to evaluate the optimized results in the case of Beijing Zoo Station in Beijing subway at last. This paper verifies the feasibility and practicability of the optimization model in order to propose positive suggestions not only on the design of layout of fare gates in new stations but also on the adjustment in existing stations.
\end{abstract}

\section{Introduction}

The field of pedestrian behavior modelling and simulation is the key to solve the crowd in rail transit stations [1,2]. Researches have been conducted from multiple disciplines owing to its microcosmic characteristics [3,4]. Simulation of pedestrians' walking trajectories [5] and selection tendency is an important requirement for efficient design of urban public areas like terminals and rail transit stations. Nevertheless, the inter-relationship between a passenger's selection tendency and the layout forms of fare gates is often neglected in this area of research.

In this paper, we investigate the basic layout forms of fare gates and confirm the index including the queue waiting density and the idleness of a set of fare gates, which can measure the service level of fare gates. We propose a multistage pedestrian-and-gate route choice model on account of time cost and design the corresponding algorithm. The basic idea is that we convert the time cost to the selection probability and decide which to choose according to the probability based on the random number method. Then, different layout schemes of fare gates are selected and the corresponding pedestrian simulation environment are established respectively by changing the scale of passenger flow and the layout types of the fare gate. Successful applications on Beijing Zoo Station in Beijing subway are evidences of the feasibility and practicability of the optimization model and strategy.

\section{Layout forms and evaluation system}

The station hall is the main place where passengers gather, distribute and transit. There actually exists some conflict points when the passenger flows intersect. The layout of fare gates in the station hall and the relative location to other service facilities can play a favorable role in controlling both the direction and the scale of passenger flows. In consideration of the relative location between fare gates in a group, we distinguish fare gates by judging if the orientation is parallel to or perpendicular to the passenger flows. We conclude the forms of fare gates in four types (Fig.1).

To measure the comprehensive service level of fare gates, we define $\rho$ as the waiting density in the queue. It represents the degree of crowdedness in the queue in front of fare gates. As one of the indexes which indicate the availability of the spatial queue, this variable should be measured both in centralized tendency and degree of dispersion. The average value $\mu_{1}$ is defined as the measured value to reflect the 
centralized tendency and the standard deviation $s_{1}$ is defined as the measured value to reflect the degree of dispersion. In the same way, we define $\lambda$ as the idleness of fare gates. With the purpose of assessing all the simulation schemes, we denote the eigenvalue to quantify the schemes with different layout forms and different scale of passenger flow as $E$ (Eq.1), where $i=1,2, \bar{U}_{i}$ means the average value of all $\mu_{i}$ for random $i$ and $\bar{S}_{i}$ means the average value of all $s_{i}$ for random $i$. The bigger the average value is, the better the schemes will be.

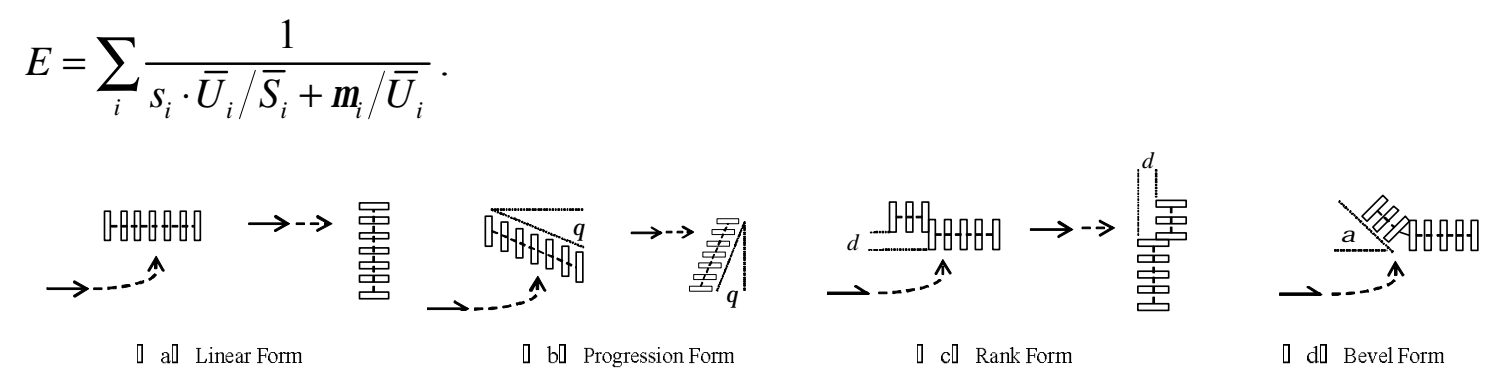

Fig.1 Different layout forms of fare gates

\section{Multistage pedestrian-and-gate route choice model}

Pace zone. Passengers always appear at the security check and walk to the fare gates to get in the station hall in the condition of different scales of flows. The walking speed would change in the zone which is from the security check to the fare gates. We divide the zone into four parts, including the general pace zone, the buffered pace zone, the go through pace zone and the regulatory pace zone according to different actions that the passengers behave in the zone. Referring to the fare gate to get out, there still exists the same pace zone. Under normal circumstances, the passengers always disperse when they get in and centralize when they get out. Affected by the thought of getting out of the station as far as possible, they always tends to walk at a higher speed. For the regulatory zone, passengers who want to get out are slower than that wants to get in. Obviously, the pressure is lower.

Selection process. The route is a selection resulting from all kinds of internal causes and external causes. The selection process is continuously changing and the indexes is also dynamic. We divided the selection process into four stages which correspond to the different pace zones. The first stage is that passengers want to get into general pace zone 1. They would decide how to walk later after judging how many people, who wants to the nearest fare gate, are waiting in general pace zone 2 . Before passengers go into general pace zone 2, they would calculate the total time comprehensively, including the waiting time because of the queue and the walking time to the fare gate. The time cost of one fare gate reflects the tendency to it. In the third stage, passengers who choose the nearest fare gate will make their third judgment after getting into general pace zone 2 . They would calculate how many people are waiting in front of the nearest fare gate. When the number is over the specific given value, they may turn to other fare gates which are far from them and change their route. In the last stage, passengers arrived at regulatory zone and swipe through the fare gate. Here, they finished the process to get in or get out.

Probabilistic algorithm based on time cost. Here is the illustration of the judging process when passengers decide which rote to choose after correction mental distance (Fig.2). The second stage of selection process is the core because it determines the final object to some extent. Here, we give an example which includes four fare gates to explain the algorithm. Note that $G_{a}\left(x_{a}, y_{a}\right), G_{b}\left(x_{b}, y_{b}\right), G_{c}\left(x_{c}, y_{c}\right), G_{d}\left(x_{d}, y_{d}\right)$ represent the coordinate of four fare gates. Let $f(x)=l_{1}$ be the boundary function between general zone 1 and 2 . Similarly, $f(x)=l_{2}$ be the boundary function between general zone 2 and buffered zone. When the passenger $i$ appears at the point $(x, 0)$, the algorithm would count the amount of passengers who want to choose the nearest fare 
gates in general zone 2. If the number is more than $m_{1}$, the object will narrow to $c$ or $d$ and the value of $p[i]$.target will be 3 or 4 . Otherwise, $a$ or $b$ will be chosen and the value will be 1 or 2 . Passengers will walk to the initial object in their original speed. When they arrive at general zone 2, they will do their second judgment on the fare gates. The algorithm would calculate the walking time to the fare gate in the current speed (time $=$ distance divided by the speed) and the time cost due to the queue in buffered zone (define the delay time of the $j$ th passenger in the queue as $p[j] . t \_p a s s$ ). The cost of the superposition is defined as the total cost spend on the selection fare gate which are denoted as $t_{a}, t_{b}, t_{c}, t_{d}$. Obviously, the smaller the cost is, the more likely the corresponding fare gate will be chosen. The eigenvalue of the fare gate is $p_{a}$ (Eq.2). The larger the eigenvalue is, the more likely the passengers will prefer to.

$$
p_{a}=1-\frac{t_{a}}{t_{a}+t_{b}+t_{c}+t_{d}}
$$

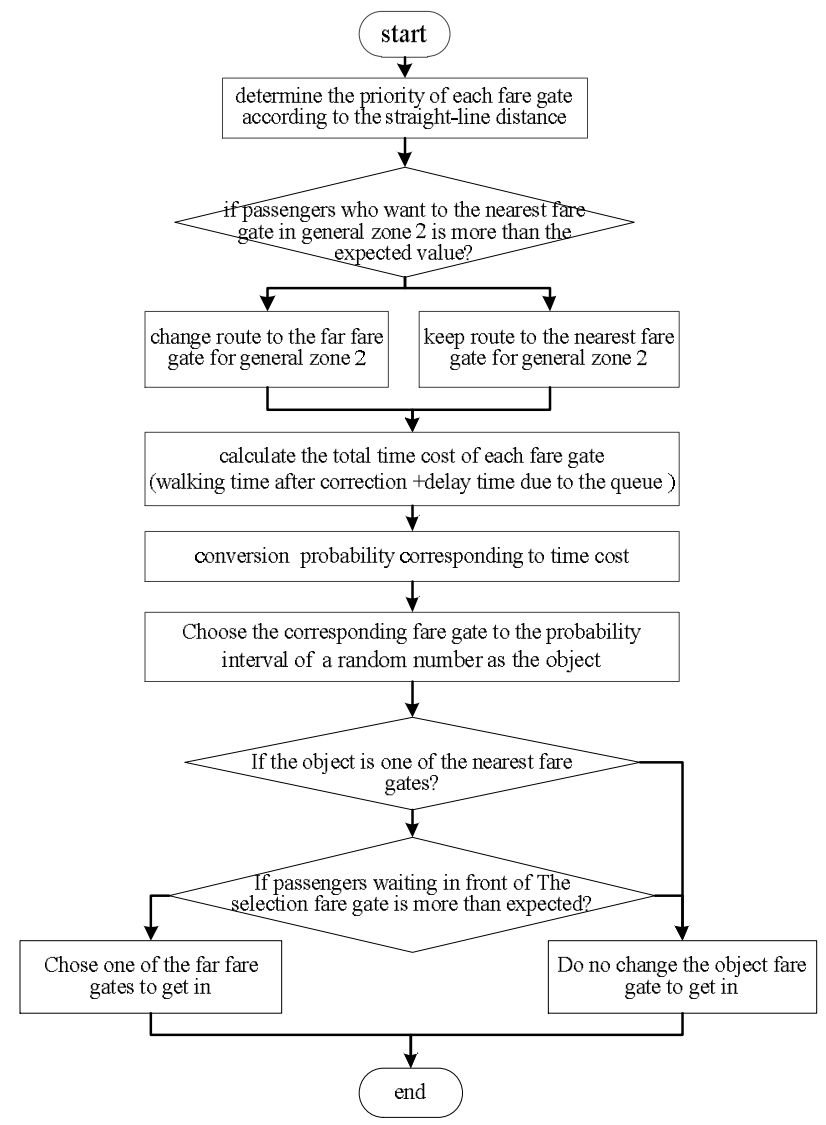

Fig.2. Illustration of probabilistic algorithm based on time cost

By building the total range and the sub range, we edit the code of random number generation between 0 and $p_{a}+p_{b}+p_{c}+p_{d}$. The sub interval where the random number is would be selected. It means the corresponding fare gate would be the current object. Then, the algorithm would generate new $p[i]$.target and $p[i] . v 2$. When the final selected fare gate is still one of the nearest fare gates $(a$ and $b)$, the algorithm would make a judgment on the amount of passengers in buffer zone. If the number is more than $m_{2}$, the object will change to $c$ and $d$. finally, the passenger get into the buffer zone in front of the selected far gate and walk in speed of $p[i] . v 3$ until to the far gate.

\section{Simulation experiment and optimization study}

Simulation experiment. In order to simulate the selection process of passengers, Anylogic7.0.2 is used to build the multistage route choice model. We choose social force model and decorate the 
selection process of pedestrians' movement. At the same time, we can obtain some statistical data. The given station here is the through type of positive convexity.

Illustrative case on Beijing Zoo Station. Here we take Beijing Zoo Station in Beijing Subway as an example. Combining with practical situation in the station, there exists an invalidation in a short time because of the fluctuation in rush hours. Practically, the passing time is larger than the theoretical value. We makes a statistical analysis on 606 service time. The average value is $2.25 \mathrm{~s}$ and $70.96 \%$ of the sample is in the range from $2 \mathrm{~s}$ to $2.5 \mathrm{~s}$. By changing the scale of passenger flow and the layout types of the fare gate, we conduct some simulation experiments and carry on data analysis. Here, we set three kinds of passengers flow in the simulation experiments. Large-scale of passengers flow is about 5000 person in an hour, medium-scale is about 2000 person in an hour and small-scale is about 1000 person in an hour. All simulation schemes are given in Table 1.

Table.1. Different simulation schemes

\begin{tabular}{|c|c|c|c|}
\hline Item & Number & Layout form & Parameter property \\
\hline \multirow{4}{*}{ Get in } & $1-9$ & Bevel-vertical & slant angle $\alpha \in\left[0,60^{\circ}\right]$ \\
\cline { 2 - 5 } & 10 & Liner-parallel & $/$ \\
\cline { 2 - 5 } & $11-12$ & Rank-vertical & recess distance $d \in\{1 \mathrm{~m}, 2 \mathrm{~m}\}$ \\
\cline { 2 - 5 } & $13-14$ & Rank-parallel & recess distance $d \in\{1 \mathrm{~m}, 2 \mathrm{~m}\}$ \\
\hline \multirow{4}{*}{ Get out } & 15 & Liner-vertical & $/$ \\
\cline { 2 - 5 } & 16 & Liner-parallel & $/$ \\
\cline { 2 - 5 } & $17-19$ & progression- vertical & slant angle $\theta \in\left[15^{\circ}, 45^{\circ}\right]$ \\
\cline { 2 - 5 } & $20-22$ & progression- parallel & slant angle $\theta \in\left[15^{\circ}, 45^{\circ}\right]$ \\
\cline { 2 - 5 } & $23-24$ & Rank-vertical & recess distance $d \in\{1 \mathrm{~m}, 2 \mathrm{~m}\}$ \\
\cline { 2 - 5 } & $25-26$ & Rank-parallel & recess distance $d \in\{1 \mathrm{~m}, 2 \mathrm{~m}\}$ \\
\hline
\end{tabular}

Result and discussion. According to the evaluation system referred in section 2 . The eigenvalue of all simulation schemes are shown in Fig.3. For fare gates in Beijing zoo station, the bevel-vertical layout form with performs better than others when passengers get in to the station. At the same time, the progression-parallel layout form with is the best. The combining scheme can get the most effect of reducing the line density. Actually, in Fig.3, the best choice is Rank-parallel. Considering the actual terrain of Beijing zoo station, it is difficult to make scheme 14 come true. So we focus our attention on bevel-vertical schemes. Here we obtain the fitted curves between and in Fig.4. It is easily to find that the scheme with performs better than others.

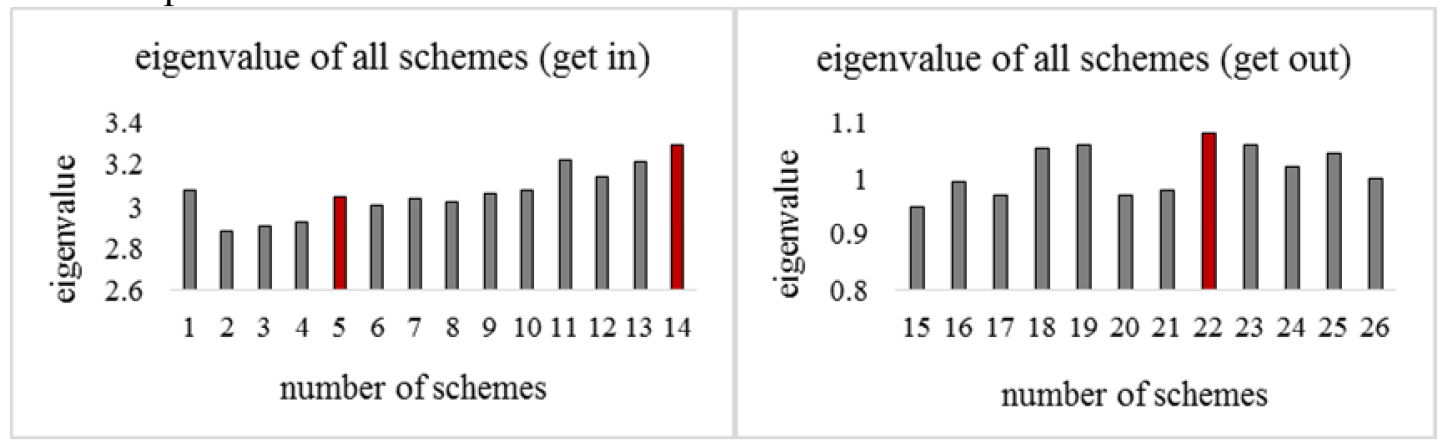

Fig.3. Eigenvalue of all schemes 


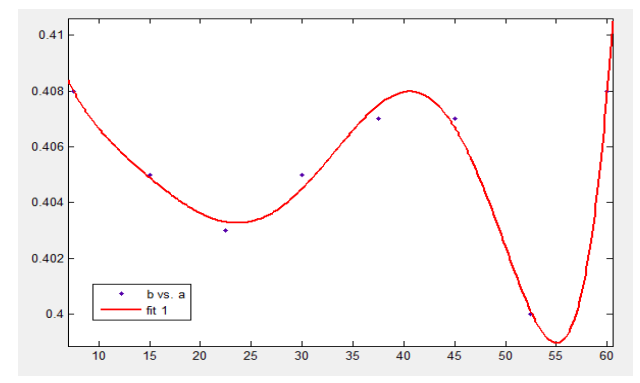

Fig.4. Fitted curves between $\alpha$ and $\lambda$

From the above simulation result, we have verified that the final optimization schemes should consider the practice in the station hall. We should take various factors into consideration, such as the passengers and the equipment. Certainly, it is significant to emphasize on only one point (the waiting density in the queue or the idleness of fare gates.) to optimize the layout of fare gates.

\section{Conclusions}

A multistage pedestrian-and-gate route choice model is able to model and predict the selection results of pedestrians within a built environment has been developed. For purpose of analyzing different layout schemes of fare gates, the corresponding pedestrian simulation environment are established respectively by changing the scale of passenger flow and the layout types of the fare gate. The algorithm is designed to carry out the selection process based on based on probabilistic time cost. The results verify the feasibility and practicability of the optimization model. It may be valuable to propose positive suggestions not only on the design of layout of fare gates in new stations but also on the adjustment in current stations.

\section{Acknowledgements}

This work was financially supported by "the Fundamental Research Funds for the Central Universities".

\section{References}

[1] R.A. Metoyer, J.K. Hodgins, Reactive pedestrian path following from examples, Visual Computer. 20 (10) (2004) 635-649.

[2] S.P. Hoogendoorn, W Daamen, Pedestrian behavior at bottlenecks [J]. Transportation Science, 2005, 39(2):147-159.

[3] M Isaacson, Application of tracking technologies to the study of pedestrian spatial behavior [J]. Professional Geographer, 2006, 58(2):172-183.

[4] J Zacharias, Pedestrian behavior and perception in urban walking environments [J]. Journal of Planning Literature: Incorporating The CPL Bibliographies, 2001, 16(1):3-18.

[5] X. Zheng, T. Zhong, M. Liu, Modeling crowd evacuation of a building based on seven methodological approaches, Build. Environ. 44 (3) (2009) 437-445. 